POS PROCEEDINGS

\title{
D-meson and charmed-baryon production in small systems with ALICE
}

Jeremy Wilkinson* for the ALICE Collaboration

Centro Fermi \& INFN Sezione di Bologna

E-mail: jeremy.wilkinson@cern.ch

This work presents the latest results on the production of charmed baryons and D mesons in proton-proton and proton-lead collisions, measured by the ALICE Collaboration at the LHC.

European Physical Society Conference on High Energy Physics - EPS-HEP2019 -

10-17 July, 2019

Ghent, Belgium

${ }^{*}$ Speaker. 


\section{Introduction}

The production of heavy-flavour particles - those containing charm or beauty quarks - is one of the leading observables in the study of the Quark-Gluon Plasma (QGP) in heavy-ion collider experiments. Due to the large masses of the charm and beauty quarks $\left(m_{\mathrm{c}, \mathrm{b}} \gg \Lambda_{\mathrm{QCD}}\right)$, they are formed only in the earliest stages of a collision from hard partonic scatterings, and not produced at later times in thermal processes. This, coupled with a lifetime longer than the freeze-out time of the QGP, means that they undergo the entire evolution of the system, and so studies of their interactions with the medium offer a unique insight into the transport properties of the QGP.

Understanding the behaviour of these particles in smaller collision systems $-\mathrm{pp}$ and $\mathrm{p}-\mathrm{Pb}-$ is crucial to our studies of the QGP. In these smaller systems, where the average particle multiplicity is much lower than in $\mathrm{Pb}-\mathrm{Pb}$, a deconfined medium is not expected to be formed. Proton-proton collisions serve as a baseline against which production rates in $\mathrm{Pb}-\mathrm{Pb}$ collisions can be compared, in order to determine whether the formation of certain species is suppressed or enhanced due to the presence of a medium. Further to this, comparisons between $\mathrm{p}-\mathrm{Pb}$ and $\mathrm{Pb}-\mathrm{Pb}$ collisions allow us to disentangle so-called "Cold Nuclear Matter" (CNM) effects - those due solely to the presence of a nucleus in the initial collision - from final-state interactions within the medium.

As well as being a baseline for heavy-ion measurements, $\mathrm{pp}$ and $\mathrm{p}-\mathrm{Pb}$ collisions are useful to study in their own right. The large masses of the charm and beauty quarks make it possible to calculate their production cross sections to a reasonable precision using perturbative QCD (pQCD) calculation frameworks. Comparisons of theory with data allow the parameters of these models to be better constrained, allowing the total charm cross section to be extracted with greater precision.

Charmed baryons are also a vital tool for testing hadronisation mechanisms for charm quarks in hadronic collisions. Previous measurements by ALICE [1,2] have shown an increase of the baryon-to-meson ratio above that predicted by models tuned to fragmentation results from $\mathrm{e}^{+} \mathrm{e}^{-}$ colliders, implying that there may be hadronisation effects such as quark recombination or colour reconnection that are present in a pp environment but not in a leptonic collision system. Precise measurements are therefore crucial to better understand these effects, and to more effectively take account of the baryon contribution to the total charm cross section.

Charmed baryons and mesons are reconstructed in ALICE at mid-rapidity in their fully hadronic decay channels: $\mathrm{D}^{0} \rightarrow \mathrm{K}^{-} \pi^{+}, \mathrm{D}^{+} \rightarrow \mathrm{K}^{-} \pi^{+} \pi^{+}, \mathrm{D}^{*+} \rightarrow \mathrm{D}^{0} \pi^{+}, \mathrm{D}_{\mathrm{s}}^{+} \rightarrow \phi \pi^{+}, \Lambda_{\mathrm{c}}^{+} \rightarrow \mathrm{pK}^{-} \pi^{+}$, and $\Lambda_{\mathrm{c}}^{+} \rightarrow \mathrm{pK}_{\mathrm{S}}^{0}$. The benefit of this approach is that there is no missing mass, meaning that the full kinematic information of the original particle is preserved in its decay products. In addition, $\Xi_{\mathcal{c}}^{0}$ baryons are reconstructed in semileptonic decays, $\Xi_{\mathrm{c}}^{0} \rightarrow \mathrm{e}^{+} \Xi^{-} v_{\mathrm{e}}$.

The ALICE detector and its performance are described in detail in [3, 4]. The Inner Tracking System (ITS) is used for the tracking of charged particles and the reconstruction of their decay vertices at mid-rapidity. This information contributes to selections made on the topology of the decay, for example the decay length and the angular separation of candidate pairs of decay daughters. Surrounding the ITS is a large gas Time Projection Chamber (TPC), which is the main tracking detector of the experiment, as well as providing particle identification (PID) information via the specific energy loss $\mathrm{d} E / \mathrm{d} x$ of charged particles passing through this. Further PID is provided by the Time of Flight (TOF) detector, which measures the flight times of charged particles. The TPC and TOF signals can be combined in order to make more distinct selections between species, in 
order to reduce contamination and therefore combinatorial background in the analysis, thereby improving the quality of the signal extraction.

\section{Results}

The production cross section of D mesons is a key component of the total charm cross section, as well as constraining the parameters of pQCD models. Another interesting observable is the relative abundance of the different $\mathrm{D}$-meson species, measured through the ratios of their production cross sections. Differential measurements of these ratios and comparisons between different collision energies and $p_{\mathrm{T}}$ regions offer a test of the universality of the fragmentation functions of charm quarks to $\mathrm{D}$ mesons. The measured $\mathrm{D}^{+} / \mathrm{D}^{0}, \mathrm{D}^{*+} / \mathrm{D}^{0}, \mathrm{D}_{\mathrm{s}}^{+} / \mathrm{D}^{0}$ and $\mathrm{D}_{\mathrm{s}}^{+} / \mathrm{D}^{+}$ratios at $\sqrt{s}=5.02 \mathrm{TeV}$ and $\sqrt{s}=7 \mathrm{TeV}$ [5] are shown in Fig. 1 (left). In all of these measurements, it can be seen that there is no significant $p_{\mathrm{T}}$ dependence of the particle ratio over the range studied, nor a dependence on the collision energy. This holds true also for $\mathrm{D}$ mesons containing a strange quark $\left(\mathrm{D}_{\mathrm{s}}^{+}\right)$, indicating that there is no momentum- or energy-dependent effect on the hadronisation introduced by the presence of a strange quark.
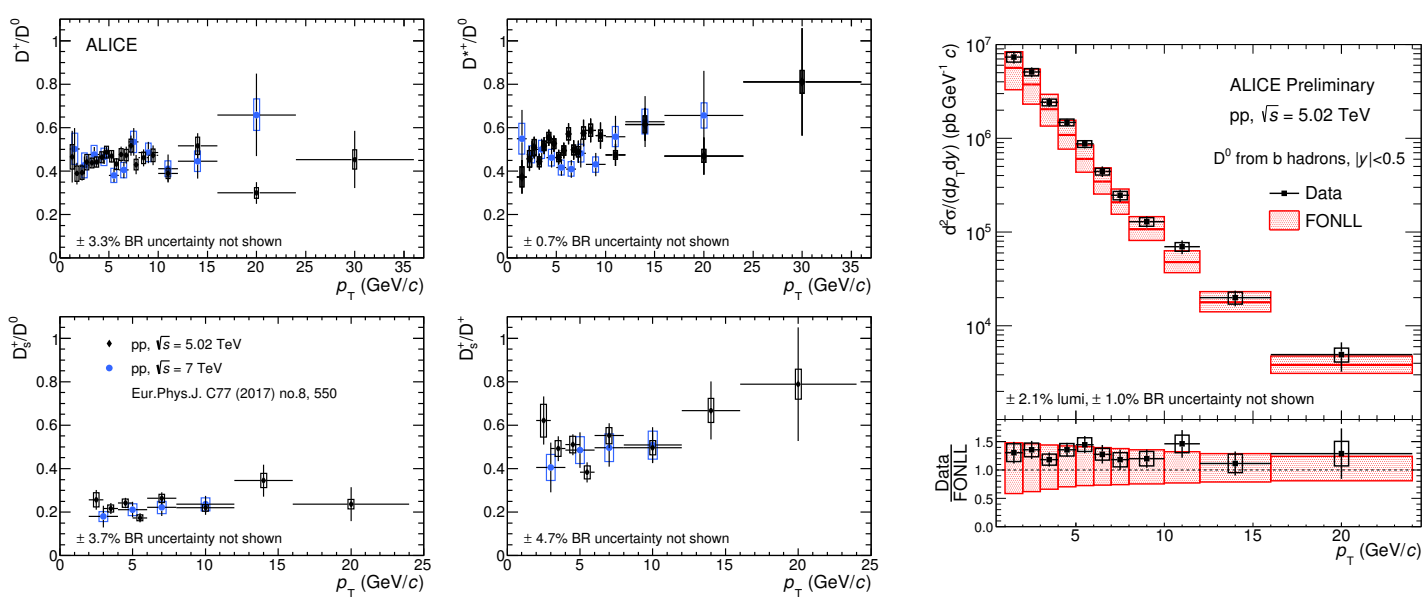

Figure 1: Left: ALICE measurement of relative D-meson production ratios in pp collisions at $\sqrt{s}=5.02 \mathrm{TeV}$ and $\sqrt{s}=7 \mathrm{TeV}$ [5]. Right: Preliminary measurement of the differential production cross section of nonprompt $\mathrm{D}^{0}$ mesons, compared with FONLL [6, 7] pQCD calculations.

Thanks to the high resolution of the ALICE tracking detectors and the leverage of machine learning techniques, it is also possible to directly measure the non-prompt contribution to D-meson production. This indirectly gives access to the b-quark production cross section in ALICE, as the non-prompt component comes from the feed-down from decays of beauty hadrons. The measurement was performed using the Boosted Decision Tree (BDT) method offered by the Toolkit for Multivariate Data Analysis (TMVA) [8] package. The $p_{\mathrm{T}}$-differential production cross section of non-prompt $\mathrm{D}^{0}$ mesons is shown in Fig. 1 (right), where it is compared against pQCD calculations using the FONLL framework $[6,7]$. The data are consistent with the FONLL predictions within the theoretical uncertainties; however, it can also be seen that the results systematically occupy the upper region of the uncertainty band. This observation is consistent with what was seen in previ- 
ous ALICE measurements of prompt charmed mesons [9,5], possibly indicating that the central parameters on the prediction should be re-tuned to better match the data.

The measurement of $\mathrm{D}$ mesons in $\mathrm{p}-\mathrm{Pb}$ collisions allows the modification of particle spectra due to $\mathrm{CNM}$ effects to be disentangled from that due to final-state in-medium interactions in $\mathrm{Pb}-\mathrm{Pb}$ collisions. The main observable is the nuclear modification factor, $R_{\mathrm{pPb}}$, which is computed by dividing the $p_{\mathrm{T}}$-differential production cross section in $\mathrm{p}-\mathrm{Pb}$ collisions by that obtained in pp collisions, and rescaling by the mass of the $\mathrm{Pb}$ nucleus $(A=208)$. A deviation of this value from unity would imply either an enhancement (above 1) or suppression (below 1) of particle production as compared to simple binary nucleon-nucleon collisions. The latest ALICE results for the $R_{\mathrm{pPb}}$ of prompt non-strange D mesons at mid-rapidity [10] are shown in Fig. 2 (left). For $p_{\mathrm{T}}>1 \mathrm{GeV} / c$, the measurement is the average of $\mathrm{D}^{0}, \mathrm{D}^{+}$and $\mathrm{D}^{*+}$ mesons, which were consistent with one another within the uncertainties and therefore averaged to improve the precision; for $p_{\mathrm{T}}<1 \mathrm{GeV} / c$ only the $\mathrm{D}^{0}$ meson is considered. The $R_{\mathrm{pPb}}$ value is consistent with unity over the entire $p_{\mathrm{T}}$ range, implying that the suppression of non-strange $\mathrm{D}$ mesons observed in $\mathrm{Pb}-\mathrm{Pb}$ collisions [11] is purely a final-state effect.

The $\mathrm{p}-\mathrm{Pb}$ system can be studied in a more differential manner by considering the $Q_{\mathrm{pPb}}-$ this is calculated in the same way as the $R_{\mathrm{pPb}}$, but sliced into intervals of centrality. The $Q_{\mathrm{pPb}}$ results in five centrality classes are shown in Fig. 2 (right) [10] and compared with the equivalent results for light-flavour charged particles. The results for the D mesons remain consistent with unity in all centralities, and are also consistent within uncertainties with the charged particles, with only a hint of an increasing trend towards more central events. This indicates that there is no significant centrality dependence of CNM effects for D mesons, and also no significant flavour dependence for these effects in general.
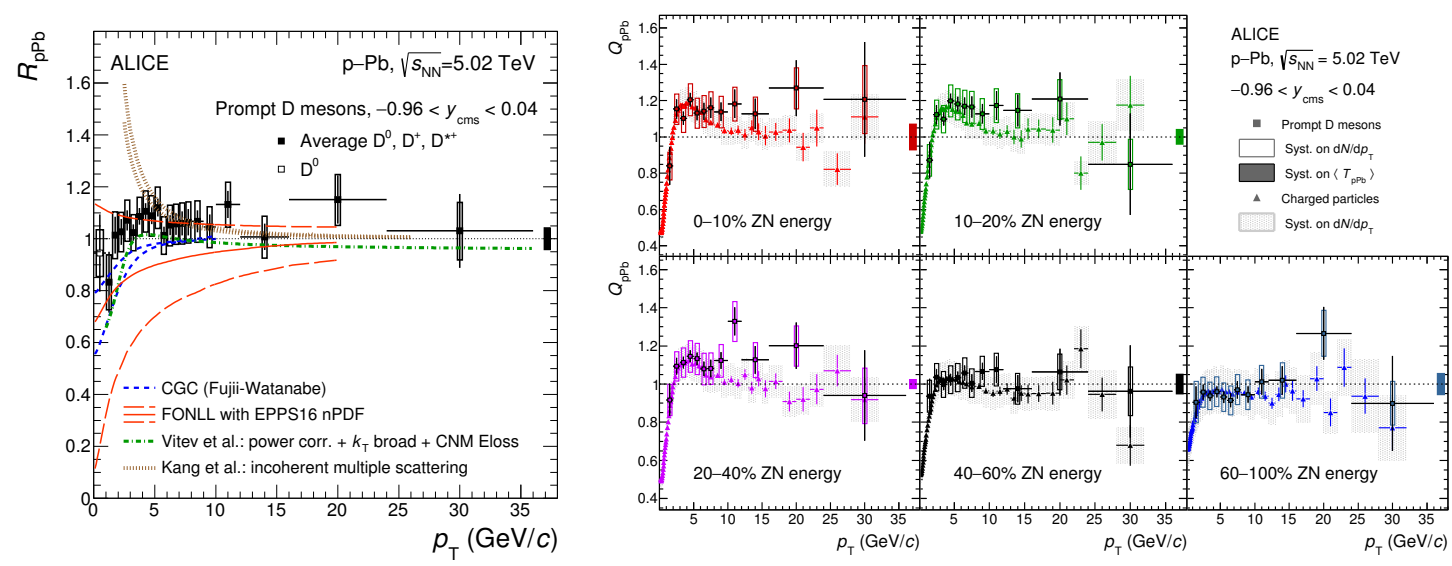

Figure 2: Left: Nuclear modification factor, $R_{\mathrm{pPb}}$, of $\mathrm{D}$ mesons in $\mathrm{p}-\mathrm{Pb}$ collisions at $\sqrt{s_{\mathrm{NN}}}=5.02 \mathrm{TeV}$, compared with model calculations. Right: $Q_{\mathrm{pPb}}$ of D mesons in five centrality classes, compared with equivalent ALICE measurements of charged particles. [10]

In the charmed baryon sector, the most accessible candidate is the $\Lambda_{\mathrm{c}}^{+}$baryon. This is measured in pp and $\mathrm{p}-\mathrm{Pb}$ collisions using both the $\Lambda_{\mathrm{c}}^{+} \rightarrow \mathrm{pK}^{-} \pi^{+}$and $\Lambda_{\mathrm{c}}^{+} \rightarrow \mathrm{pK}_{\mathrm{S}}^{0}$ decay channels; the measured cross sections were consistent between both channels, and therefore are averaged to enhance the precision of the result. For the $\Lambda_{\mathrm{c}}^{+} \rightarrow \mathrm{pK}_{\mathrm{S}}^{0}$ analysis in $\mathrm{p}-\mathrm{Pb}$ collisions, a fur- 
ther machine learning technique using BDTs was applied as an alternative approach to reduce the background. The $R_{\mathrm{pPb}}$ of $\Lambda_{\mathrm{c}}^{+}$baryons is shown in Fig. 3. In the left panel, it is compared against the equivalent measurement for $\mathrm{D}$ mesons; within the statistical and systematic uncertainties, both measurements are consistent with one another. In addition, the results are compared with POWHEG+PYTHIA6 Monte Carlo calculations [12], which include only CNM effects, and the POWLANG transport model [13], which simulates the formation of a small QGP within the $\mathrm{p}-\mathrm{Pb}$ collision. The POWLANG curve appears to be favoured by the comparison with data at lower $p_{\mathrm{T}}$; however, neither model is able to fully describe the measurement at all $p_{\mathrm{T}}$.

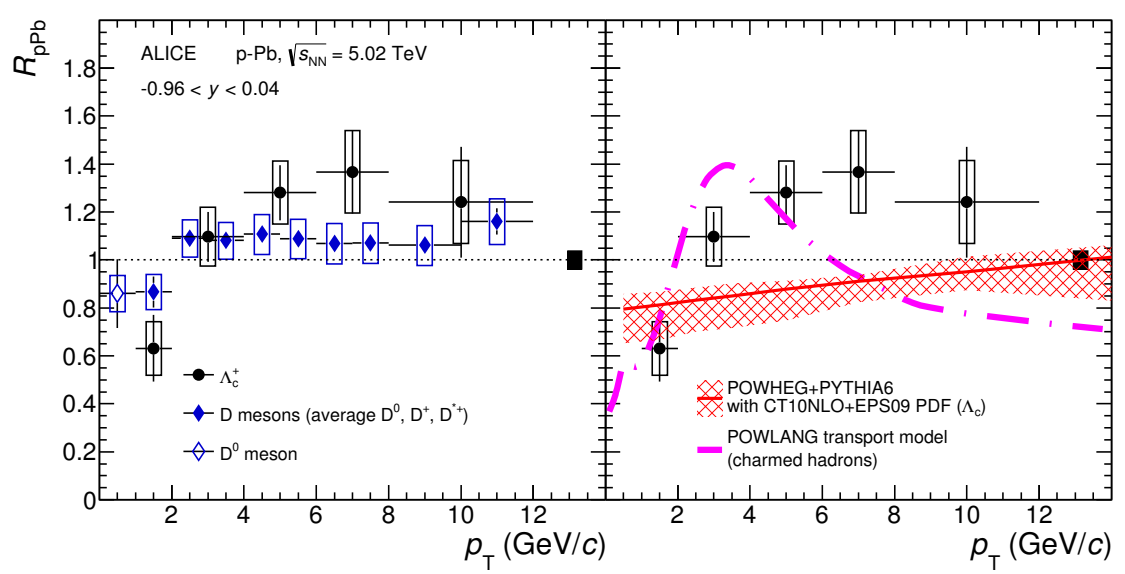

Figure 3: Nuclear modification factor $R_{\mathrm{pPb}}$ of $\Lambda_{\mathrm{c}}^{+}$baryons in $\mathrm{p}-\mathrm{Pb}$ collisions at $\sqrt{s_{\mathrm{NN}}}=5.02 \mathrm{TeV}$. Left: Compared with ALICE measurement of D mesons. Right: Compared with model calculations.

The key observable in baryon measurements is the baryon-to-meson ratio. For charmed baryons, the $\Lambda_{\mathrm{c}}^{+} / \mathrm{D}^{0}$ ratio is used, defined as the ratio between the $p_{\mathrm{T}}$-differential cross sections of the two species. In Fig. 4 (left), this ratio is compared with the light-flavour baryon-to-meson ratios, $\Lambda / \mathrm{K}_{\mathrm{S}}^{0}$ and $\mathrm{p} / \pi$, for both $\mathrm{pp}$ and $\mathrm{p}-\mathrm{Pb}$ collisions. All three ratios in both systems exhibit similar behaviour, with the indication of a peak around $p_{\mathrm{T}}=3 \mathrm{GeV} / c$, and in particular the charmed $\Lambda_{\mathrm{c}}^{+} / \mathrm{D}^{0}$ ratio is very closely consistent with the strangeness sector $\left(\Lambda / \mathrm{K}_{\mathrm{S}}^{0}\right)$. We also compare the $\Lambda_{\mathrm{c}}^{+} / \mathrm{D}^{0}$ ratio directly between $\mathrm{p}-\mathrm{Pb}, \mathrm{Pb}-\mathrm{Pb},(\mathrm{pp})$ collisions at $\sqrt{s_{\mathrm{NN}}}=5.02 \mathrm{TeV}(\sqrt{s}=5.02 \mathrm{TeV})$ in Fig. 4 (right), where a hint of a hierarchy can be seen at intermediate $p_{\mathrm{T}}$ when going from smaller systems (pp) to larger ones (central $\mathrm{Pb}-\mathrm{Pb}$ collisions); however, further measurements with better precision would be required in all systems in order to confirm this.

Comparisons of the $\Lambda_{\mathrm{c}}^{+} / \mathrm{D}^{0}$ ratio against Monte Carlo calculations can be seen in Fig. 5 (left). It can be seen that the curves without enhanced colour reconnection (Monash, DIPSY, HERWIG7), which are tuned to fragmentation results from $\mathrm{e}^{+} \mathrm{e}^{-}$collisions, significantly underestimate the $\Lambda_{\mathrm{c}}^{+} / \mathrm{D}^{0}$ ratio, implying that the hadronisation mechanisms for charm quarks differ between leptonic and hadronic collision systems. This would also have implications for calculations of the total charm production cross section, which use existing fragmentation measurements in order to extract the value from the cross sections of individual species. The PYTHIA8 SoftQCD model comes closest to reproducing the data, reflecting the broad shape of the $\Lambda_{\mathrm{c}}^{+} / \mathrm{D}^{0}$ ratio as a function of $p_{\mathrm{T}}$, although is still systematically below the data points.

Finally, we consider the production of higher charmed baryon states such as the $\Xi_{\mathrm{c}}^{0}$ [14]. This 

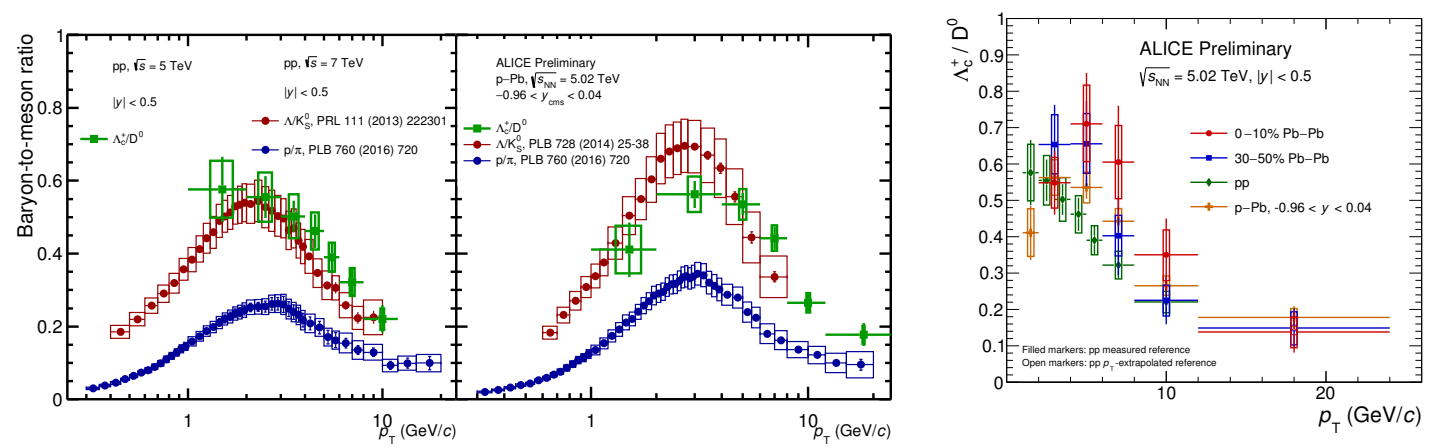

Figure 4: Left: Nuclear modification factor, $R_{\mathrm{pPb}}$, of $\mathrm{D}$ mesons in $\mathrm{p}-\mathrm{Pb}$ collisions at $\sqrt{s_{\mathrm{NN}}}=5.02 \mathrm{TeV}$, compared with model calculations. Right: $Q_{\mathrm{pPb}}$ of $\mathrm{D}$ mesons in five centrality classes, compared with equivalent ALICE measurements of charged particles. [10]
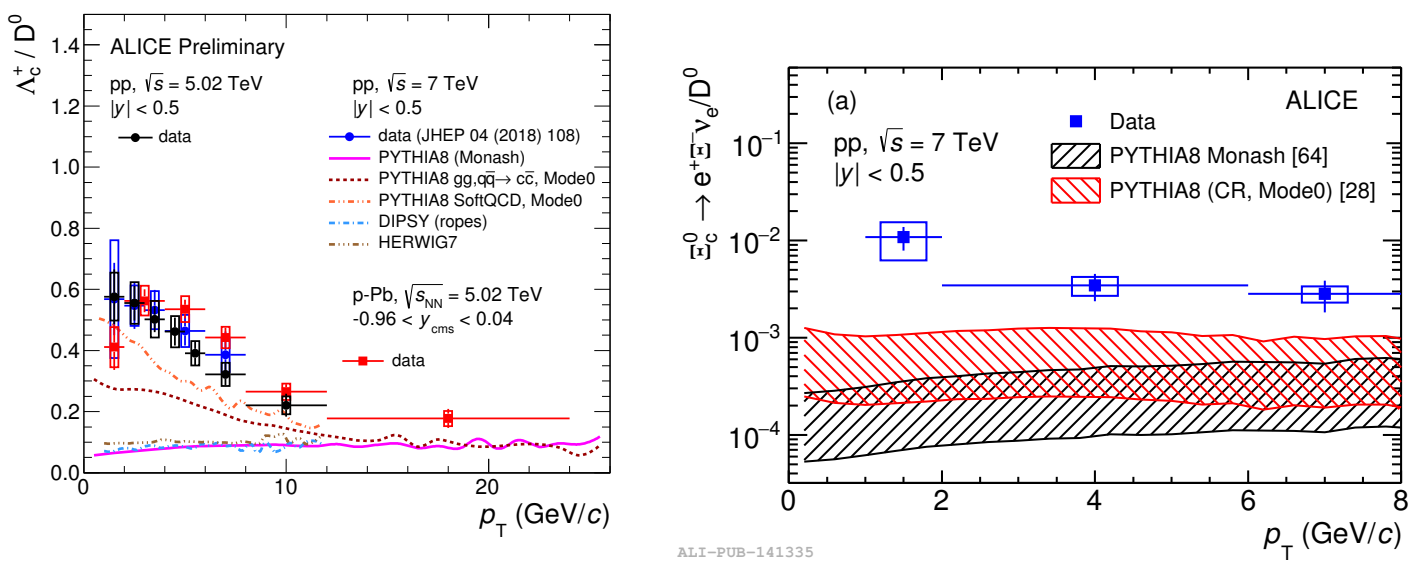

Figure 5: Left: $\Lambda_{\mathrm{c}}^{+} / \mathrm{D}^{0}$ baryon-to-meson ratio for $\mathrm{pp}$ and $\mathrm{p}-\mathrm{Pb}$ collisions, compared with models. Right: $\Xi_{\mathrm{c}}^{0} / \mathrm{D}^{0}$ baryon-to-meson ratio in pp collisions at $\sqrt{s}=7 \mathrm{TeV}$, compared with PYTHIA Monte Carlo predictions [14]

baryon contains both a charm and a strange quark, and so allows us to see whether the presence of both of these flavours together modifies the rate of baryon hadronisation. Currently in ALICE this species is measured in its semileptonic decay channel. As the absolute branching fraction is unknown, we present the cross section multiplied by the semileptonic branching ratio. This is shown in Fig. 5 (right) in the form of the $\Xi_{\mathrm{c}}^{0} / \mathrm{D}^{0}$ baryon-to-meson ratio, compared with PYTHIA Monte Carlo calculations using two different tunes. As was seen for the $\Lambda_{\mathrm{c}}^{+}$baryons, the MC predictions significantly underestimate the observed results, and so further study is required in order to better understand the effects at play for this species.

\section{Outlook}

Charmed hadron production in small systems remains one of the key observables for ALICE at the LHC. Run 2 of the LHC provided a wealth of data which is still being examined. In particular, the full Run 2 sample of pp collisions at $\sqrt{s}=13 \mathrm{TeV}$ will allow for even more precise 
measurements of the $\mathrm{D}$ mesons, as well as allowing for differential studies of their production as a function of the charged-particle multiplicity.

One of the key challenges in the charm sector has proven to be the reproduction of the measured charmed baryon-to-meson ratios by model calculations. Further measurements with high precision, and understanding on the theory side, will be needed in order to better explain the observed differences between the hadronisation ratios in hadronic and leptonic collisions. As part of this, it will be instructive to look into the $\Xi_{c}^{0}$ and $\Xi_{c}^{+}$baryons in their hadronic decay channels, which will allow a better reconstruction of their decay kinematics. The $\Sigma_{\mathrm{c}}^{0}$ charm baryon resonance, which is expected to provide significant feed-down for the $\Lambda_{\mathrm{c}}^{+}$measurement, provides another interesting line of study for these effects.

Finally, the upgraded ITS and TPC in Run 3 of the LHC, as well as the increase in luminosity provided by the collider, will allow for much more precise measurements of charm production in all collision systems in ALICE. In addition, it will become possible to make direct measurements of beauty production in hadronic decays in ALICE.

\section{References}

[1] ALICE Collaboration, $\Lambda_{\mathrm{c}}^{+}$production in pp collisions at $\sqrt{s}=7 \mathrm{TeV}$ and in $p$-Pb collisions at $\sqrt{s_{\mathrm{NN}}}=5.02 \mathrm{TeV}$, JHEP 04 (2018) 108 [1712.09581].

[2] ALICE Collaboration, $\Lambda_{\mathrm{c}}^{+}$production in Pb-Pb collisions at $\sqrt{s_{\mathrm{NN}}}=5.02 \mathrm{TeV}$, Phys. Lett. B793 (2019) 212 [1809.10922].

[3] ALICE Collaboration, The ALICE experiment at the CERN LHC, JINST 3 (2008) S08002.

[4] ALICE Collaboration, Performance of the ALICE Experiment at the CERN LHC, Int. J. Mod. Phys. A29 (2014) 1430044 [1 402.4476$].$

[5] ALICE Collaboration, Measurement of $\mathrm{D}^{0}, \mathrm{D}^{+}, \mathrm{D}^{*+}$ and $\mathrm{D}_{\mathrm{s}}^{+}$production in pp collisions at $\sqrt{s}=5.02 \mathrm{TeV}$ with ALICE, Eur. Phys. J. C79 (2019) 388 [1901. 07979].

[6] M. Cacciari, S. Frixione, N. Houdeau, M. L. Mangano, P. Nason and G. Ridolfi, Theoretical predictions for charm and bottom production at the LHC, JHEP 10 (2012) 137 [1205.634 4].

[7] M. Cacciari, M. L. Mangano and P. Nason, Gluon PDF constraints from the ratio of forward heavy-quark production at the LHC at $\sqrt{S}=7$ and $13 \mathrm{TeV}$, Eur. Phys. J. C75 (2015) 610 [1507.06197].

[8] A. Höcker, P. Speckmayer, J. Stelzer, J. Therhaag, E. von Toerne and H. Voss, TMVA: Toolkit for Multivariate Data Analysis, PoS ACAT (2007) 040 [physics / 0703039 ].

[9] ALICE Collaboration, D-meson production in p-Pb collisions at $\sqrt{s_{\mathrm{NN}}}=5.02 \mathrm{TeV}$ and in $p p$ collisions at $\sqrt{s}=7$ TeV, Phys. Rev. C94 (2016) 054908 [1605.07569].

[10] ALICE Collaboration, Measurement of prompt $D^{0}, D^{+}, D^{*+}$, and $D_{s}^{+}$production in $p-P b$ collisions at $\sqrt{s_{\mathrm{NN}}}=5.02 \mathrm{TeV}, 1906.03425$.

[11] ALICE Collaboration, Measurement of $D^{0}, D^{+}, D^{*+}$ and $D_{s}^{+}$production in $\mathrm{Pb}-\mathrm{Pb}$ collisions at $\sqrt{\mathrm{S}_{\mathrm{NN}}}=5.02 \mathrm{TeV}$, JHEP 10 (2018) 174 [1804.09083].

[12] S. Frixione, P. Nason and G. Ridolfi, A Positive-weight next-to-leading-order Monte Carlo for heavy flavour hadroproduction, JHEP 09 (2007) 126 [0707. 3088]. 
[13] A. Beraudo, A. De Pace, M. Monteno, M. Nardi and F. Prino, Heavy-flavour production in high-energy d-Au and p-Pb collisions, JHEP 03 (2016) 123 [1512. 05186 ].

[14] ALICE Collaboration, First measurement of $\Xi_{\mathrm{c}}^{0}$ production in pp collisions at $\sqrt{\mathbf{s}}=7 \mathrm{TeV}$, Phys. Lett. B781 (2018) 8 [1712.04242]. 